\title{
Abordagem do paciente em primeiro episódio de surto psicótico: revisão de literatura
}

\author{
Approach to the patient in the first episode of a psychotic episode: literature review
}

Abordaje del paciente en el primer episodio de un episodio psicótico: revisión de la literatura

Recebido: 16/09/2021 | Revisado: 22/09/2021 | Aceito: 24/11/2021 | Publicado: 26/11/2021

\author{
Maria Thais Caldas Araújo \\ ORCID: https://orcid.org/0000-0002-4661-9760 \\ Faculdade Santa Maria, Brasil \\ E-mail: thaiscaldascalu@gmail.com \\ Marcus Vinicius Dutra dos Santos \\ ORCID: https://orcid.org/0000-0001-8236-4614 \\ Universidade Federal do Rio Grande do Norte, Brasil \\ E-mail: marcusdutras@gmail.com \\ Marina Gentil Heraclio Maia \\ ORCID: https://orcid.org/0000-0001-9069-9510 \\ Faculdade Santa Maria, Brasil \\ E-mail: marinamaia_mm@hotmail.com \\ Renan Marques Gadelha Rodrigues \\ ORCID: https://orcid.org/0000-0001-8674-3910 \\ Faculdade Santa Maria, Brasil \\ E-mail: renangadelha@yahoo.com.br \\ Carmem Raquel Marques Coura Aragão \\ ORCID: https://orcid.org/0000-0002-4307-1488 \\ Faculdade Santa Maria, Brasil \\ E-mail: carmemrmca@gmail.com \\ Maria Luiza Abreu Pessoa \\ ORCID: https://orcid.org/0000-0003-2627-5304 \\ Faculdade Santa Maria, Brasil \\ E-mail: mluizaabreup@gmail.com \\ Lillian Rodrigues Farias \\ ORCID: https://orcid.org/0000-0001-7344-1550 \\ Faculdade Santa Maria, Brasil \\ E-mail: lillian-rodrigues@ hotmail.com \\ Maria Beatriz Cruz Macedo \\ ORCID: https://orcid.org/0000-0002-4900-8762 \\ Faculdade Santa Maria, Brasil \\ E-mail: biacruzmac@gmail.com \\ José de Arimateia Oliveira Santiago \\ ORCID: https://orcid.org/0000-0001-6501-3927 \\ Faculdade Santa Maria, Brasil \\ E-mail: arimateioliveirasantiago@ @otmail.com \\ João Marcos Batista Gomes de Araujo \\ ORCID: https://orcid.org/0000-0001-6877-2179 \\ Faculdade Santa Maria, Brasil \\ E-mail: joaomarcosbg@hotmail.com
}

\begin{abstract}
Resumo
As encelopatias são constituídas por um grupo de etiologias heterogênicas, tendo uma condição clínica que necessita de rápida atenção para serem identificadas. Dentre as encelopatias, algumas vem sendo mais estudadas nas últimas décadas, como a Encefalopatia de Hashimoto (EH), doença foi descrita em 1966 por Jellinek como uma patologia cerebral com componente vascular e presença de anticorpos anti-tiroideus, em que pode estar relacionada com Tiroidite de Hashimoto (TH). Essa doença pode manifestar-se em episódios semelhantes a acidentes vasculares cerebrais, síndromes psiquiátricas e ataques epilépticos em $66 \%$ dos casos e não tem características paraclínicas típicas. Além disso, é resistente à fármacos e envolve uma possível progressão para apreensões parcialmente complexas, raramente generalizadas, possivelmente seguidas de fraqueza. Nesse contexto, o presente artigo tem como objetivo verificar as principais repercussões fisiopatológicas e clínicas da Encefalopatia de Hashimoto a partir de um estudo bibliográfico e de caráter exploratório, utilizando descritores validados por meio dos Descritores em Ciências da Saúde (DECS). Verificou-se que há fatores relacionados com a doença, como a predominância genética, carência de elementos químicos. Além disso, estudos indicaram que resultados das imagens electroencefalográficas e de ressonância magnética são consistentes na detecção da encefalopatia de Hashimoto.
\end{abstract}

Palavras-chave: Encefalopatia de Hashimoto; Tireoidite de Hashimoto; Sinais clínicos. 


\begin{abstract}
Encelopathies are constituted by a group of heterogeneous etiologies, having a clinical condition that needs rapid attention to be identified. Among the encelopathies, some have been more studied in recent decades, such as Hashimoto's Encephalopathy (HE), a disease that was described in 1966 by Jellinek as a brain pathology with a vascular component and presence of anti-thyroid antibodies, which may be related to Hashimoto's thyroiditis (TH). This disease can manifest itself in stroke-like episodes, psychiatric syndromes, and epileptic seizures in $66 \%$ of cases and does not have typical paraclinical features. Furthermore, it is drug resistant and involves a possible progression to partially complex, rarely generalized seizures, possibly followed by weakness. In this context, this article aims to verify the main pathophysiological and clinical repercussions of Hashimoto's Encephalopathy from a bibliographical and exploratory study, using descriptors validated through Health Sciences Descriptors (DECS). It was found that there are factors related to the disease, such as genetic predominance, lack of chemical elements. Furthermore, studies have indicated that electroencephalographic and magnetic resonance imaging results are consistent in detecting Hashimoto's encephalopathy.
\end{abstract}

Keywords: Hashimoto's Encephalopathy; Hashimoto's Thyroiditis; Clinical signs.

\title{
Resumen
}

Las encelopatías están constituidas por un grupo de etiologías heterogéneas, teniendo una condición clínica que requiere atención inmediata para ser identificada. Entre las encelopatías, algunas han sido más estudiadas en las últimas décadas, como la Encefalopatía de Hashimoto (EH), enfermedad que fue descrita en 1966 por Jellinek como una patología cerebral con componente vascular y presencia de anticuerpos antitiroideos, que pueden estar relacionados a la tiroiditis de Hashimoto $(\mathrm{TH})$. Esta enfermedad puede manifestarse en episodios similares a ictus, síndromes psiquiátricos y ataques epilépticos en el $66 \%$ de los casos y no tiene características paraclínicas típicas. Además, es resistente a los fármacos e implica una posible progresión a convulsiones parcialmente complejas, rara vez generalizadas, posiblemente seguidas de debilidad. En este contexto, este artículo tiene como objetivo verificar las principales repercusiones fisiopatológicas y clínicas de la Encefalopatía de Hashimoto a partir de un estudio bibliográfico y exploratorio, utilizando descriptores validados a través de Descriptores de Ciencias de la Salud (DECS). Se encontró que existen factores relacionados con la enfermedad, como predominio genético, falta de elementos químicos. Además, los estudios han indicado que los resultados de las imágenes electroencefalográficas y de resonancia magnética son consistentes en la detección de la encefalopatía de Hashimoto.

Keywords: Encefalopatía de Hashimoto; Tiroiditis de Hashimoto; Signos clínicos.

\section{Introdução}

As encelopatias são constituídas por um grupo de etiologias heterogênicas, tendo uma condição clínica que necessita de rápida atenção para serem identificadas (Nascimento, 2018). Segundo o autor, podem ocorrer por diversas formas, como crises hipertensivas, distúrbios metabólicos, traumas ou acidentes, neoplasias, infecções, doenças isquêmicas, entre outras, lesionando o cérebro e alterando suas funções.

Dentre as encelopatias, algumas vem sendo mais estudadas nas últimas décadas, como a Encefalopatia de Hashimoto (EH). De acordo com Oliveira et al. (2017), essa doença foi descrita em 1966 por Jellinek et al. (1996) como uma patologia cerebral com componente vascular e presença de anticorpos anti-tiroideus, em que pode estar relacionada com Tiroidite de Hashimoto (TH).

Gavriliuc et al. (2013) afirmam que essa doença pode manifestar-se em episódios semelhantes a acidentes vasculares cerebrais, síndromes psiquiátricas e ataques epilépticos em $66 \%$ dos casos (comumente mioclónico) e não tem características paraclínicas típicas. Além disso, os autores complementam ao afirmar que é resistente à fármacos e envolve uma possível progressão para apreensões parcialmente complexas, raramente generalizadas, possivelmente seguidas de fraqueza.

Entretanto, Oliveira et al. (2017), o mecanismo da doença não é confirmado pela literatura e diversos especialistas afirmam que estas duas patologias não estão necessariamente relacionadas, logo, por isso, Encefalopatia de Hashimoto é uma Encefalopatia respondedora a corticoides relacionada a tiroidite auto-imune e meningoencefalite auto-imune não vasculítica.

Gregory, Nóbrega Junior e Lin (2019), afirmam que hoje em dia, o diagnóstico da Encefalopatia de Hashimoto é oneroso e dependentes de testes de autoanticorpos e pouco disponibilizados nos hospitais brasileiros. As formas de diagnosticar a Encefalopatia de Hashimoto são: 1) Punção lombar; 2) Eletroencefalografia (EEG); 3) Imagem de Ressonância 
Magnética e; 4) Exames laboratoriais para as causas usuais de delirium (Garrido, 2014). O prognóstico da EH quando é tratado, é bom, 90\% dos pacientes mostram remissão após 10 anos (Gavriliuc et al., 2013).

De acordo com Damiani et al. (2013), grande parte dos pacientes responde a corticoterapia em doses elevadas. Para os autores, a terapia preconizada tem sido a administração de prednisona (1-2 mg/kg/ dia) por curto período. Entretanto, alguns pacientes precisam de tratamento prolongado para remissão dos sintomas. A melhora clínica está presente em aproximadamente 4-6 semanas após o início do tratamento.

Nesse contexto, o presente artigo tem como objetivo verificar as principais repercussões fisiopatológicas e clínicas da Encefalopatia de Hashimoto a partir de um estudo bibliográfico e de caráter exploratório.

\section{Metodologia}

\subsection{Tipo da pesquisa}

Conforme caracteriza Köche (2016), do ponto de vista da natureza, esse trabalho trata de uma pesquisa aplicada. Pela perspectiva de abordagem, é uma pesquisa qualitativa, ou seja, "são utilizados quando se quer medir opiniões, reações, sensações, hábitos e atitudes etc. de um universo (público-alvo)", como exemplos: entrevistas pessoais; entrevistas por telefone; através de cartas; questionário estruturado fechado; questionário semiestruturados e perguntas abertas; com apresentação de cartões, objetos e material promocional (Manzato \& Santos, 2012, p. 7).

Analisando os objetivos essa pesquisa é exploratória, isto é, seu objetivo é proporcionar maior familiaridade com o problema, tornar-se explicito ou construir hipóteses com seu respeito ou causar aprimoramento do tema (Pereira et al., 2018). Em relação aos procedimentos técnicos, trata-se de uma revisão bibliográfica. Pizzanin et al. (2012) afirmam que uma revisão de literatura possui diversos objetivos, como: a) proporcionar um aprendizado sobre uma determinada área do conhecimento; b) facilitar a identificação e seleção dos métodos e técnicas a serem utilizados pelo pesquisador; c) oferecer subsídios para a redação da introdução e revisão da literatura e redação da discussão do trabalho científico.

\subsection{Método}

Os procedimentos metodológicos de um trabalho acadêmico é um processo lógico com finalidade principal de atingir um determinado fim ou para se chegar ao conhecimento de um determinado assunto. O método científico consiste em um sentido mais amplo é a ordem que se deve impor aos vários processos necessários para atingir um objetivo ou resultado desejado. Já o "Método científico é um instrumento de que se serve a inteligência para descobrir relações, verdades e leis referentes aos diversos objetos de investigação" (Santos \& Parra Filho, 2012, p. 3).

Para a realização desse estudo, a primeira etapa foi a organização do problema a ser pesquisado, para posteriormente avaliar e aplicar todo o máximo do material bibliográfico disponível, uma vez que o tema deve conter relevância tanto teórica como prática e proporcionar interesse de ser estudado. Seguindo esse raciocínio, para que se possa atingir o destino cogitado, a pesquisa será dividida em três fases principais, como pode ser verificado na Figura 1. 
Figura 1: Fases que serão utilizadas no presente estudo.

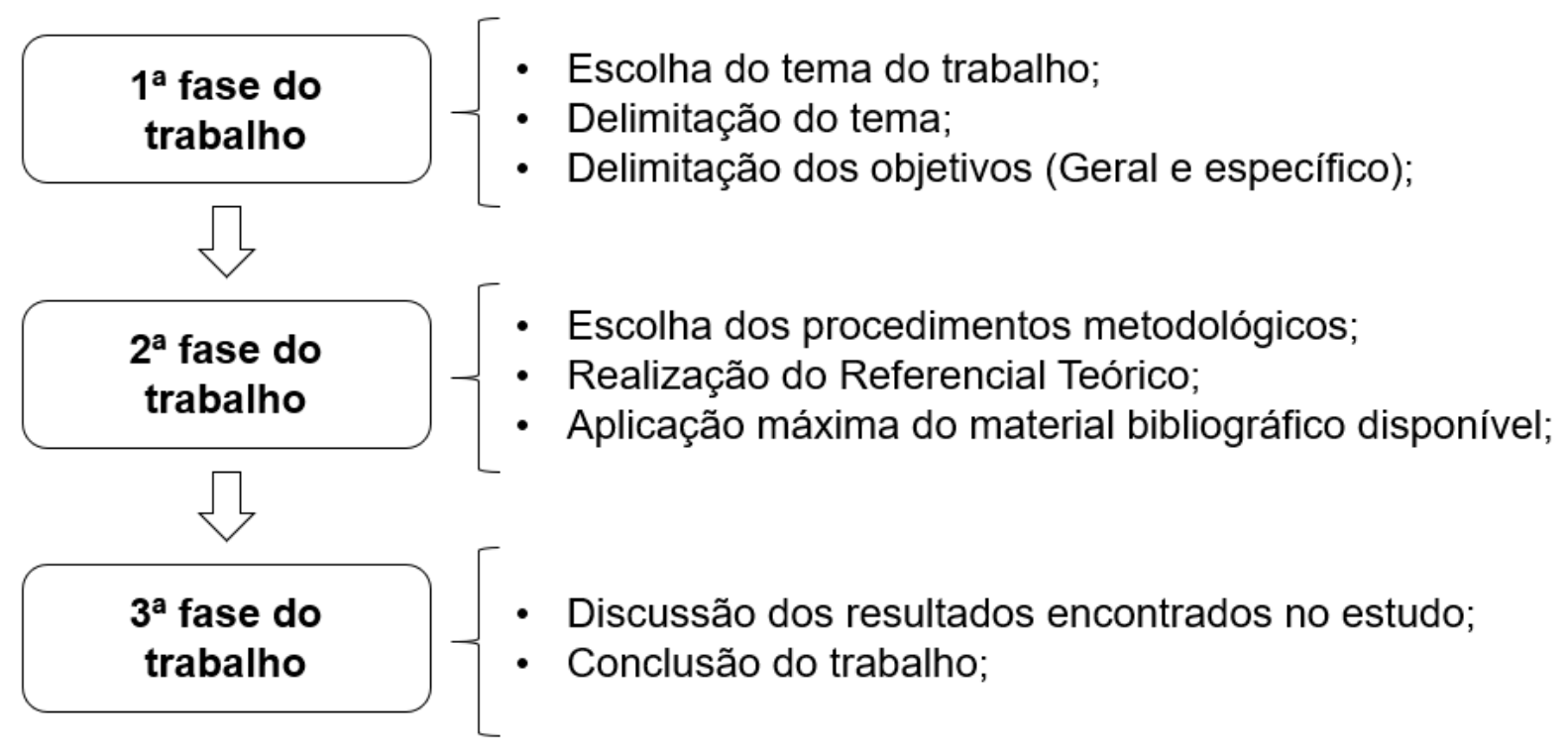

Fonte: Autores (2021).

Na presente pesquisa se utilizou descritores validados por meio dos Descritores em Ciências da Saúde (DECS). O DeCS é um vocabulário estruturado hierarquicamente, ou seja, uma árvore conceitual e terminológica que mapeia o conhecimento das ciências da saúde de modo a apresentá-lo de forma organizada, dividindo-o em categorias e subcategorias e, dentro delas, os descritores do mais geral ao mais específico (Campanatti-Ostiz \& Andrade, 2010). Os descritores utilizados foram: "Síndrome"; "Encefalopatia" e "Encefalopatia de Hashimoto".

A pesquisa foi realizada na base de dados da Biblioteca Virtual de Saúde (BVS), em que estão indexadas também outras bibliotecas virtuais, tais como SciELO (Scientific Electronic Library Online), BIRENE (Biblioteca Regional de Medicina), BDENF (Base de dados de enfermagem), LILACS (Literatura Latino-Americana e do Caribe em Ciências da Saúde), MEDLINE (Literatura Internacional em Ciências da Saúde), Coleciona SUS (Coleção Nacional das Fontes de Informação do SUS), MS (Acervo da Biblioteca do Ministério da Saúde), SAÚDE LEGIS (Legislação Federal da Saúde), OPAS (Acervo da Biblioteca da Organização Pan-Am. Saúde), WHOLIS (Sistema de Informação da Biblioteca da OMS).

A ordem de prioridade para a escolha de trabalho foi: (i) artigos publicados em periódicos internacionais; (ii) artigos publicados em periódicos nacionais reconhecidos; (iii) livros publicados por bons editores; (iv) teses e dissertações; (v) anais de conferências internacionais; (vi) anais de conferências nacionais.

No que se refere a delimitação temporal, o presente trabalho realizará uma pesquisa sobre o tema nos últimos 10 anos. Já no que se refere os Critérios de exclusão: artigo noticiosos, textos em resenhas, artigos não indexados, opiniões, editoriais ou manuais. Além disso, foi adotada para a análise de dados a Análise Temática de Conteúdo, informações que segundo Minayo (2010), desdobra-se nas etapas pré-análise, exploração do material ou codificação e tratamento dos resultados obtidos/ interpretação.

Para análise e interpretação dos dados do presente estudo, foi utilizado o software Excel para realizar a tabulação dos dados encontrados, bem como a elaboração de tabelas apresentando os principais resultados encontrados. Com base nesta pesquisa e na análise foi possível estabelecer uma linha do tempo do tema da pesquisa, de forma evolutiva, que resultará nas pesquisas mais recentes. 


\section{Resultados e Discussões}

As encefalopatias associadas à tireoidite de Hashimoto não são comuns (foram descritos cerca de 200 casos até 2018 : a prevalência é de cerca de 2/1.000.000), no entanto, acomete predominantemente mulheres (aproximadamente 90\% dos casos descritos na literatura afetam o sexo feminino), com quarenta e quatro anos, em média (Irigaray et al., 2011). Os autores complementam ao afirmar que os sintomas neurológicos são os seguintes: alteração do nível da consciência, sinais focais, crises epilépticas, estado confusional, mioclonias, ataxias, tremores, desordens mentais, demência e adulterações motoras.

A partir da seleção dos artigos do tema de interesse desse estudo, verificou há fatores relacionados com a doença, como a predominância genética, carência de elementos químicos. O Quadro 1 apresenta os principais resultados obtidos nesse estudo. Os resultados desta pesquisa geraram um conjunto inicial de documentos, que foram filtrados os resultados iniciais da pesquisa a partir da leitura do título e do resumo. Todos os artigos encontrados, relacionados ao tema, foram incluídos na análise, independentemente de ser o assunto principal do artigo ou apenas mencionado no resumo. Para organizar as informações dos trabalhos selecionados da base dados, foi utilizada a leitura flutuante dos títulos e resumos dos trabalhos bem como os resultados apresentados.

Quadro 1: Resultados obtidos no presente estudo.

\begin{tabular}{|c|c|c|c|}
\hline Autores & Título & Objetivo & Resultados \\
\hline $\begin{array}{c}\text { Sur et al. } \\
\text { (2019) }\end{array}$ & $\begin{array}{l}\text { Marcadores de stress } \\
\text { oxidativos, elementos } \\
\text { vestigiais e químicos } \\
\text { desreguladores } \\
\text { endócrinos em crianças } \\
\text { com tireoidite de } \\
\text { Hashimoto }\end{array}$ & $\begin{array}{l}\text { Investigar se a exposição ao bisfenol A } \\
\text { (BPA) e ao ftalato de di-(2-etil-hexilo) } \\
\text { (DEHP) tem alguma associação com a } \\
\text { tiroidite de Hashimoto (HT) e os seus } \\
\text { biomarcadores e determinar se os } \\
\text { biomarcadores de stress oxidativo e os } \\
\text { níveis de oligoelementos mostraram } \\
\text { alguma alteração nas crianças com HT. }\end{array}$ & $\begin{array}{l}\text { Não foi comprovada a relação entre o estresse oxidativo e } \\
\text { a Tireoidite de Hashimoto. Entretanto, os níveis de zinco } \\
\text { foram significativamente mais baixos no grupo de HT vs. } \\
\text { controlo. Além disso, os níveis de ftalato mono-( } 2 \text { - } \\
\text { etilhexil) (MEHP), que é o metabolito primário do DEHP, } \\
\text { foram marcadamente mais elevados no grupo HT em } \\
\text { comparação com o controlo (p < 0,05). Foi observada uma } \\
\text { correlação negativa entre os níveis de BPA urinário e fT4. } \\
\text { Em crianças com HT, o equilíbrio oxidante/antioxidante é } \\
\text { alterado e estas diferenças podem estar relacionadas com a } \\
\text { exposição a EDC. }\end{array}$ \\
\hline $\begin{array}{l}\text { Kawasaki } \\
\text { et al. } \\
(2019)\end{array}$ & $\begin{array}{l}\text { A associação entre o } \\
\text { equilíbrio hormonal da } \\
\text { tiróide e o volume da } \\
\text { tiróide em doentes com } \\
\text { tiroidite Hashimoto }\end{array}$ & $\begin{array}{l}\text { Investigar a relação entre o estado da } \\
\text { hormonal tiroidiana e o volume da } \\
\text { tiróide (VT) entre pacientes com } \\
\text { Encefalopatia de Hashimoto }\end{array}$ & $\begin{array}{l}\text { Os resultados indicaram que os pacientes de HT com } \\
\text { aumento da TV tendiam a apresentar taxas elevadas de } \\
\text { FT3 sérica, FT4 baixas, e FT3/FT4 altas. A elevação da } \\
\text { atvidade da deiodinase pode ser um factor importante que } \\
\text { afetar o equilíbrio hormonal da tiróide em tais doentes. }\end{array}$ \\
\hline $\begin{array}{c}\text { Jeong et } \\
\text { al. (2019) }\end{array}$ & $\begin{array}{l}\text { A associação entre a } \\
\text { ecogenicidade da tiróide e } \\
\text { a função tireoidiana na } \\
\text { tiroidite de Hashimoto } \\
\text { pediátrica e adolescente }\end{array}$ & $\begin{array}{l}\text { Avaliar a associação entre a } \\
\text { ecogenicidade da tiróide na ultra- } \\
\text { sonografia (EUA) e a função da tiróide } \\
\text { em doentes pediátricos e adolescentes } \\
\text { com tiroidite de Hashimoto }(\mathrm{HT}) \text {. }\end{array}$ & $\begin{array}{c}\text { Verificaram que nos pacientes pediátricos e adolescentes } \\
\text { Encefalopatia de Hashimoto, houve uma associação entre } \\
\text { a diminuição da ecogenicidade e disfunção da tiróide e } \\
\text { níveis elevados de TSH, mesmo em sujeitos com função } \\
\text { tiroideia normal, ou hipotiroidismo subclínico ou } \\
\text { explícito. Sugerindo que os EUA podem ser úteis para } \\
\text { complementar os ensaios bioquímicos na avaliação do } \\
\text { estado da tiróide. São necessárias mais investigações com } \\
\text { estudos de acompanhamento para avaliar a utilidade dos } \\
\text { EUA para prever a progressão da função tireoidiana } \\
\text { anormal. }\end{array}$ \\
\hline $\begin{array}{l}\text { Mijajlovic } \\
\text { et al. } \\
(2010)\end{array}$ & $\begin{array}{l}\text { Manifestações clínicas, } \\
\text { critérios diagnósticos e } \\
\text { terapia da encefalopatia } \\
\text { de Hashimoto: Relato de } \\
\text { dois casos }\end{array}$ & $\begin{array}{c}\text { Verificar a partir de relato de caso as } \\
\text { manifestações clínicas, critérios } \\
\text { diagnósticos e terapia da encefalopatia } \\
\text { de Hashimoto }\end{array}$ & $\begin{array}{l}\text { Verificaram através dos casos relatados duas formas } \\
\text { distintas emergiram: um tipo vasculítico caracterizado por } \\
\text { múltiplos episódios de recidivas-remitentes e uma ligeira } \\
\text { deficiência cognitiva e um tipo progressivo difuso } \\
\text { caracterizado por demência e sintomas psiquiátricos. } \\
\text { Ambas as formas podem ser acompanhadas por nível de } \\
\text { consciência deprimido, tremor, convulsões ou mioclonos. }\end{array}$ \\
\hline $\begin{array}{l}\text { Gul Mert } \\
\text { et al. } \\
(2014)\end{array}$ & $\begin{array}{l}\text { A encefalopatia de } \\
\text { Hashimoto: quatro casos } \\
\text { e revisão de literatura }\end{array}$ & $\begin{array}{c}\text { Descrever os resultados clínicos e } \\
\text { laboratoriais de quatro crianças (com 8- } \\
17 \text { anos de idade) com a encefalopatia } \\
\text { de Hashimoto. }\end{array}$ & $\begin{array}{l}\text { As características clínicas de três pacientes na } \\
\text { apresentação incluíram epilepsia refratária, e confusão, e } \\
\text { um paciente apresentado com alterações comportamentais } \\
\text { e cognitivas. Durante a sua apresentação, dois deles } \\
\text { estavam em eutróide, e os outros estavam em estado } \\
\text { hipotiróide. Todos os doentes manifestaram aumento de } \\
\text { anticorpos anti-tiróides. Dois doentes melhoraram com o } \\
\text { tratamento com esteróides. Os outros reagiram à } \\
\text { plasmaferese em vez do tratamento com corticosteróides }\end{array}$ \\
\hline
\end{tabular}




\begin{tabular}{|c|c|c|c|}
\hline $\begin{array}{l}\text { Canelo- } \\
\text { Aybar et } \\
\text { al. }(2010)\end{array}$ & $\begin{array}{c}\text { A encefalopatia de } \\
\text { Hashimoto apresenta } \\
\text { sintomas neurocognitivos: } \\
\text { um relato de caso }\end{array}$ & $\begin{array}{l}\text { Apresentar um relato de caso sobre as } \\
\text { repercussões clínicas de paciente com } \\
\text { Encefalopatia de Hashimoto }\end{array}$ & $\begin{array}{l}\text { A paciente era uma mulher hispânica de } 62 \text { anos de idade, } \\
\text { que desenvolveu um início subagudo de declínio da } \\
\text { função cerebral superior. Estudos serológicos } \\
\text { demonstraram níveis elevados de anticorpos anti-tiróides. } \\
\text { Os resultados das imagens electroencefalográficas e de } \\
\text { ressonância magnética foram consistentes com a } \\
\text { encefalopatia de Hashimoto. Essa paciente apresentou } \\
\text { diversos sintomas neurocognitivos e resultados normais } \\
\text { em vários exames diferentes. Esta síndrome pode não ser } \\
\text { reconhecida durante muito tempo; por conseguinte, deve } \\
\text { ser tida em conta ao avaliar um doente com disfunção } \\
\text { cognitiva e títulos elevados de anticorpos anti-tiróides. }\end{array}$ \\
\hline $\begin{array}{l}\text { Rayman } \\
\text { et al. } \\
(2019)\end{array}$ & $\begin{array}{l}\text { Factores nutricionais } \\
\text { múltiplos e doença da } \\
\text { tiróide, com particular } \\
\text { referência à doença auto- } \\
\text { imune da tiróide }\end{array}$ & $\begin{array}{l}\text { Investigar o efeito do iodo, ferro e } \\
\text { selénio no risco, patogénese e } \\
\text { tratamento da doença }\end{array}$ & $\begin{array}{l}\text { Os pacientes são frequentemente deficitários em ferro, } \\
\text { visto que reduz a absorção de ferro e a doença celíaca que } \\
\text { causa a perda de ferro, são co-morbilidades frequentes. } \\
\text { Em dois terços das mulheres com sintomas persistentes de } \\
\text { hipotiroidismo, apesar da terapia apropriada com } \\
\text { levothyroxina, a restauração da ferritina sérica acima de } \\
100 \mu \mathrm{g} / \mathrm{l} \text { apresenta sintomas melhorados. Selénio: as } \\
\text { selenoproteínas são essenciais para a acção da tiróide. }\end{array}$ \\
\hline $\begin{array}{l}\text { Bilge et } \\
\text { al. (2019) }\end{array}$ & $\begin{array}{l}\text { Razão neutrofílica e } \\
\text { plaquetária para linfócitos } \\
\text { em doentes com Tiroidite } \\
\text { de Hashimoto Eutróide }\end{array}$ & $\begin{array}{l}\text { Investigar a relação entre a } \\
\text { automunidade da tiróide e a NLR e a } \\
\text { PLR como marcadores de inflamação } \\
\text { sistémica no HT. }\end{array}$ & $\begin{array}{l}\text { Os resultados demostraram uma contagem inferior de } \\
\text { linfócitos, enquanto que a contagem de plaquetas, NLR, e } \\
\text { PLR foram superiores em comparação com o grupo de } \\
\text { controlo (p<0,001 para todas as comparações). Ou seja, a } \\
\text { PLR eram mais elevadas em doentes de Encefalopatia de } \\
\text { Hashimoto do que num grupo de controlo saudável. A } \\
\text { NLR e a PLR são provavelmente indicadores não } \\
\text { específicos de desordem imunológica e as suas implicaçõe } \\
\text { e outras condições ainda não foram elucidadas. }\end{array}$ \\
\hline $\begin{array}{l}\text { Önalan e } \\
\text { Dönder } \\
(2020)\end{array}$ & $\begin{array}{c}\text { Razão neutrofílica e } \\
\text { plaquetária para linfócitos } \\
\text { em doentes com tiroidite } \\
\text { de Hashimoto } \\
\text { hipotiroidismo }\end{array}$ & $\begin{array}{l}\text { Investigar a razão neutrofílica e } \\
\text { plaquetária para linfócitos em doentes } \\
\text { com tiroidite de Hashimoto }\end{array}$ & $\begin{array}{l}\text { Verificaram que os doentes diagnosticados demonstraram } \\
\text { uma NLR mais elevada e uma PLR mais baixa em } \\
\text { comparação com o grupo de controlo saudável com } \\
\text { significado estatístico, em conformidade com estudos } \\
\text { anteriores da literatura. O HT é meramente um processo } \\
\text { inflamatório que é iniciado pela estimulação dos linfócitos } \\
\text { T pelo sistema auto-imune, e embora pensemos que o } \\
\text { aumento da RNB poderia estar relacionado com os } \\
\text { mecanismos semelhantes envolvidos neste processo, } \\
\text { acreditamos que os baixos níveis de RNB. }\end{array}$ \\
\hline $\begin{array}{l}\text { Meyer } \\
\text { Neto et al. } \\
(2013)\end{array}$ & $\begin{array}{c}\text { Rara associação entre } \\
\text { linfoma não-Hodgkin e } \\
\text { encefalopatia de } \\
\text { Hashimoto: relato de caso }\end{array}$ & $\begin{array}{c}\text { Apresentar um caso de doença } \\
\text { incomum do sistema nervoso central, a } \\
\text { encefalopatia de Hashimoto }(\mathrm{EH})\end{array}$ & $\begin{array}{l}\text { Verificou que a doença pode ser observada em pacientes } \\
\text { acometidos com: distúrbios do sono, cefaleia, déficits } \\
\text { sensitivos e motores com lateralização e mesmo quadros } \\
\text { de psicose e de paranoia }\end{array}$ \\
\hline
\end{tabular}

Fonte: Autores (2021).

Meyer Neto et al. (2013) descreve que também podem ser observados em pacientes acometidos pela doença, como: distúrbios do sono, cefaleia, déficits sensitivos e motores com lateralização e mesmo quadros de psicose e de paranoia. Além disso, o curso da doença pode ser autolimitado, redicivante-remitente ou progressivo. Cozzi (2018) afirma que a patogenia ainda é desconhecida, mas acredita-se que seja autoimune, com estes mecanismos de hipóteses: vasculite autoimune do sistema nervoso central (SNC); Reatividade cruzada de autoanticorpos entre a tiróide e o SNC; feito tóxico do TRH no CNS.

Irigaray et al. (2011) afirmam que há dois tipos característicos de apresentação clínica, que podem ser sobrepostos: 1) tipo vasculítico de EH, caracterizado por episódios vasculares pseudoictais, no qual podem resultar em déficits neurológicos focais de forma aguda ou subaguda; 2) Formadifusa progressiva da EH, é caracterizado por declínio cognitivo progressivo, podendo levar a demência ou alterações psiquiátricas, como depressão, alucinações, confusão mental, sonolência e coma.

Perri, Gastaldi e Maghnie (2019) complementam ao afirmar que, provavelmente, os anticorpos antitireoidianos não sejam os únicos agentes patogênicos, pois há diversos estudos afirmando que a Encefalopatia de Hashimoto pode ser um transtorno imunomediado, em vez de representar o efeito direto de um estado alterado da tireoide. Waliszewska-Prosół e Ejma (2016) afirmam que a encefalopatia de Hashimoto pode ser caracterizada como um processo inflamatório com atributos de uma vasculite grave. 


\section{Considerações Finais}

O objetivo do presente artigo foi verificar as principais repercussões fisiopatológicas e clínicas da Encefalopatia de Hashimoto. Logo, verifica-se que o objetivo do trabalho foi cumprindo, visto que foi verificado na literatura. Verificou-se que há fatores relacionados com a doença, como a predominância genética, carência de elementos químicos.

Averiguou-se que essa doença pode manifestar-se em episódios semelhantes a acidentes vasculares cerebrais, síndromes psiquiátricas e ataques epilépticos em $66 \%$ dos casos e não tem características paraclínicas típicas. Além disso, é resistente à fármacos e envolve uma possível progressão para apreensões parcialmente complexas, raramente generalizadas, possivelmente seguidas de fraqueza.

Espera-se que o presente artigo de caráter exploratório corrobore com a literatura existente e sirva como base para futuros trabalhos sobre o tema. Como sugestão para futuros trabalhos, recomenda-se uma revisão sistemática sobre as causas da doença.

\section{Referências}

Bilge, Muge et al. (2019). Neutrophil-and platelet-to lymphocyte ratio in patients with euthyroid hashimoto's thyroiditis. Experimental and Clinical Endocrinology \& Diabetes, 127(8).

Brasil. (1998). Lei $n^{o}$.9.610, de 19 de fevereiro de 1998. http://www. planalto.gov.br/ccivil_03/leis/L9610.htm

Campanatti-Ostiz, H., \& Andrade, C. R. F. de. (2010). Descritores em Ciências da Saúde na área específica da Fonoaudiologia Brasileira. Pró-Fono Revista de Atualização Científica, v. 22.

Canelo-Aybar, Carlos et al. (2010). Hashimoto's encephalopathy presenting with neurocognitive symptoms: a case report. Journal of medical case reports, 4(1).

Cavalcante, R. B., Calixto, P., \& Pinheiro, M. M. K. (2014). Análise de conteúdo: considerações gerais, relações com a pergunta de pesquisa, possibilidades e limitações do método. Informação \& Sociedade: Estudos, 24(1).

Cozzi, R. (2018). L’encefalopatia Di Hashimoto Responsabile Editoriale. In: Ame News, 1(1).

Garrido, J. V. M. (2014). Demência rapidamente progressiva: diagnóstico diferencial. 2014. 43 f. Dissertação de Mestrado - Universidade da Beira Interior.

Gil, A. C. (2010). Como elaborar projetos de pesquisa. (5a ed.), Atlas.

Gregory, C. P., Nóbrega Jr, A. W., \& Lin, K. (2019). Encefalites autoimunes-abordagem prática para situações de recursos limitados. Boletim do Curso de Medicina da UFSC, 5(2).

Gul Mert, G. et al. (2014). Hashimoto's encephalopathy: four cases and review of literature. International Journal of Neuroscience, 124(4).

Irigaray, T. Q. et al. (2011). Avaliação neuropsicológica na encefalopatia de Hashimoto: Um relato de caso. Revista Neuropsicologia Latinoamericana, 3(1).

Jellinek, E. H. et al. (1966). Hashimoto's disease and encephalopathy. The Lancet, 288(7462).

Jeong, S. H., Hong, H. S., \& Lee, Ji Ye. (2019). The association between thyroid echogenicity and thyroid function in pediatric and adolescent Hashimoto's thyroiditis. Medicine, $98(14)$.

Kawasaki, M. et al. (2019). The association between thyroid hormone balance and thyroid volume in patients with Hashimoto thyroiditis. Endocrine jornal.

Köche, J. C. (2016). Fundamentos de metodologia científica. Editora Vozes.

Lakatos, E M., \& Marconi, M. de A. (2017). Metodologia do Trabalho Científico. (8a ed.), Atlas.

Manzato, A. J., \& Santos, A. B. (2012). A elaboração de questionários na pesquisa quantitativa. Departamento de Ciência de Computação e EstatísticaIBILCE-UNESP.

Meyer Neto, J. G. C. et al. (2013). Rara associação entre linfoma não-Hodgkin e encefalopatia de Hashimoto: relato de caso. Rev. Soc. Bras. Clín. Méd, 11(2).

Mijajlovic, M. et al. (2010). Clinical manifestations, diagnostic criteria and therapy of Hashimoto's encephalopathy: report of two cases. Journal of the neurological sciences, v. 288.

Nascimento, L. E. (2018). Santos. Estudo por imagem das encefalopatias e neurocisticercose em Hospital Público no município de Santos-SP, Brasil. UNILUS Ensino e Pesquisa, 15(39)

Oliveira, I. et al. (2017). Encefalopatia de hashimoto: uma causa de demência reversível. Revista Clínica do Hospital Prof. Doutor Fernando Fonseca, 5(2). 
Research, Society and Development, v. 10, n. 15, e567101520689, 2021

(CC BY 4.0) | ISSN 2525-3409 | DOI: http://dx.doi.org/10.33448/rsd-v10i15.20689

Pereira, A. S. et al. (2018). Metodologia da pesquisa científica. 2018. https://repositorio.ufsm.br/handle/1/15824.

Perri, K. G. R., \& Maghnie, M. (2019). Encefalopatia di Hashimoto. L'Endocrinologo, 20(2).

Pizzani, L. et al. (2012). A arte da pesquisa bibliográfica na busca do conhecimento. RDBCI: Revista Digital de Biblioteconomia e Ciência da Informação, $10(2)$.

Praça, F. S. G. (2015). Metodologia da pesquisa científica: organização estrutural e os desafios para redigir o trabalho de conclusão. Revista Eletrônica "Diálogos Acadêmicos, 8(1).

Rayman, M. P. (2019). Multiple nutritional factors and thyroid disease, with particular reference to autoimmune thyroid disease. Proceedings of the Nutrition Society, 78(1).

Santos, J. A., \& Parra Filho, D. (2012). Metodologia científica. In: Núcleo de Pós-Graduação e Extensão - FAVENI.

Sur, U. et al. (2019). Oxidative stress markers, trace elements, and endocrine disrupting chemicals in children with Hashimoto's thyroiditis. Toxicology mechanisms and methods, 29(9).

Waliszewska-Prosół, M., \& Ejma, M. (2016). Encefalopatia Hashimoto-patogeneza, obraz kliniczny i leczenie. Polski Przegląd Neurologiczny, 12(4). 